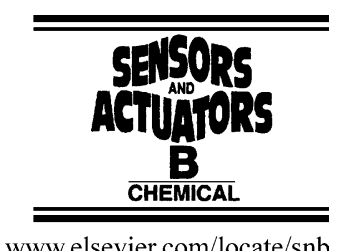

www.elsevier.com/locate/snb

\title{
Fluid modelling of microfluidic separator channels
}

\author{
R.J. Townsend ${ }^{\mathrm{a}}$, M. Hill ${ }^{\mathrm{a}, *}$, N.R. Harris ${ }^{\mathrm{b}}$, N.M. White $^{\mathrm{b}}$, S.P. Beeby ${ }^{\mathrm{b}}$, R.J.K. Wood ${ }^{\mathrm{a}}$ \\ ${ }^{a}$ Mechanical Engineering School of Engineering Science, University of Southampton, Southampton SO17 1BJ, UK \\ ${ }^{\mathrm{b}}$ ECS, University of Southampton, Southampton SO17 1BJ, UK
}

Available online 25 April 2005

\begin{abstract}
In a microfluidic ultrasonic separator device, the microchannel geometry is inherently angular due to the anisotropic etch processes used. A CFD model is used to investigate eddy formation induced by this angular geometry. Results are applicable to various microfluidic devices and show that certain etch patterns significantly reduce eddy formation.
\end{abstract}

(C) 2005 Elsevier B.V. All rights reserved.

Keywords: Microchannels; CFD; Ultrasonic separation; Fluid separation

\section{Introduction}

There is significant interest in exploiting the phenomenon whereby particles experience a radiation force when within an acoustic standing wave. Various devices using radiation forces have been reported which typically aim to concentrate, fractionate or manipulate particles for sensing purposes [1-4]. Here, a microengineered ultrasonic separator device (Fig. 1) is being developed to manipulate particles suspended within a carrier fluid using either a half or quarter wavelength standing wave to concentrate particles along certain planes within a straight fluid channel [5-7]. Two outlets subsequently draw off either clean fluid or a particle concentrate depending on requirements.

Similar devices have been fabricated from steel and glass $[4,8]$, however for this technique to be realized on a microfluidic scale etched silicon and Pyrex are being used. This has the potential advantages of batch production and easy incorporation into microfluidic systems containing micropumps, etc. which require only very small sample sizes. However, the nature of micro-engineering fabrication techniques restricts the geometric design of the microchannels. The microchannel geometry and fluid operating conditions contribute to the fluid flow field within the device which is significant in terms

\footnotetext{
* Corresponding author.

E-mail address: m.hill@ soton.ac.uk (M. Hill).
}

of the successful operation of the device. This paper concentrates on the geometric design of the cell, and its influence on pressure losses and fluid separation where the fluid becomes separated from the channel walls creating a fluid vortex (fluid separation will subsequently be referred to as eddy flows to avoid confusion with the term 'particle separation' which describes the function of the device). Eddy formation can distort particle trajectories [9] and influences sedimentation formed on the channel walls, in turn strongly influencing the performance of the device. Therefore, a computational fluid dynamics (CFD) model has been used to investigate the effects of microchannel geometry on the fluid flow.

\section{Microchannel geometry}

\subsection{Separator construction and geometry}

The micromachined separator device consists of a $525 \mu \mathrm{m}$ thick silicon wafer and $1700 \mu \mathrm{m}$ Pyrex wafer, etched and then joined using anodic bonding. Fluid enters and leaves the device via ducts etched into the silicon. The duct geometries currently used in the separator consist of either single or double sided etched ducts that lead to an etched channel within the Pyrex across which the acoustic standing wave is set up. Fig. 2 illustrates the typical wafer geometry incorporating either single or double etched ducts. 


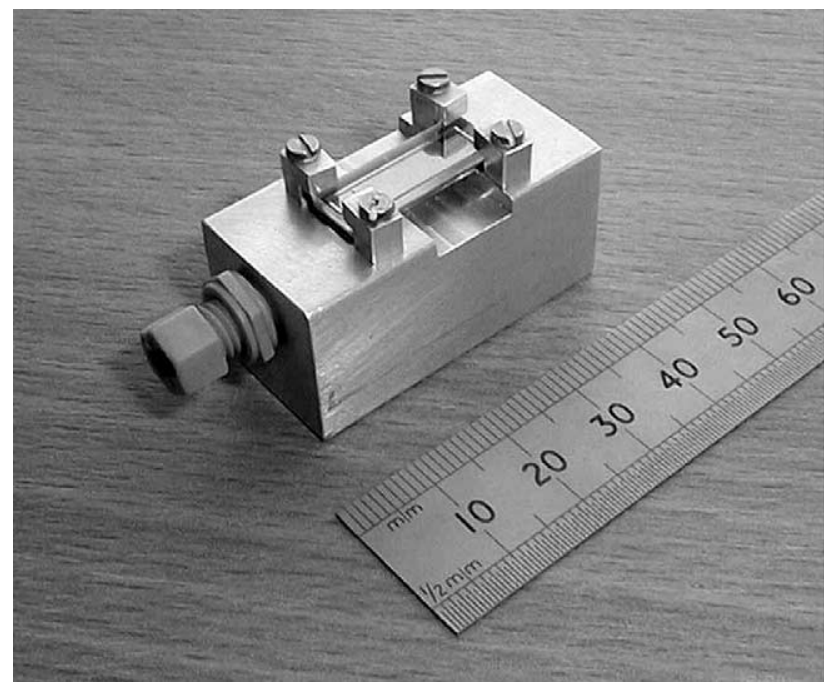

Fig. 1. Microengineered ultrasonic flow-through separator mounted on aluminium manifold.

(a)

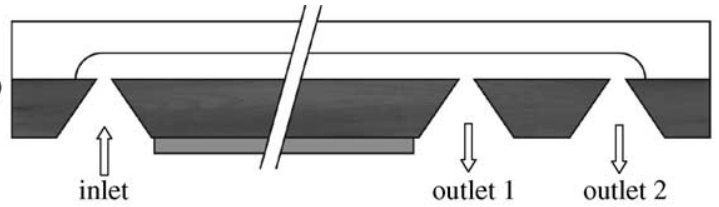

(b)

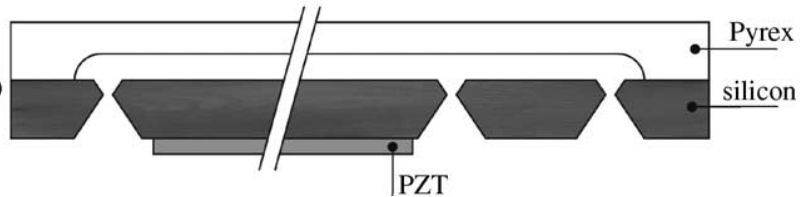

Fig. 2. Schematic of typical cross-section of device structure illustrating both (a) single and (b) double-sided etched duct geometry within the silicon wafer, with both flow direction and materials indicated.
Isotropic and anisotropic etchants are used for the Pyrex and silicon wafers respectively, where the anisotropic etch produces predictable angular geometries where the etch process is effectively halted at the (1 111 ) crystal planes, forming angles of $54.74^{\circ}$. Alternative methods of creating the ducts, such as deep ion etching, exist and although such methods may provide more geometric variations, this study reveals a satisfactory solution based simply on wet etching techniques.

\subsection{Influence of geometry on separator performance}

Where anisotropically etched geometry is dictated by the orientation of the wafer crystal planes, discontinuities in the wall geometry are created. Such discontinuities cause fluid flow to separate from the channel wall giving rise to eddy flows in which the direction of flow is reversed at the wall [10,11]. Fig. 3 presents an example of a CFD simulation used to predict eddy formation within the inlet region of the device. Fig. 3a illustrates the formation of an eddy downstream of the double-etched duct which is indicated by the reversed flow at the wall. There is also an eddy region at the end of the main channel, contained within the curved isotropic etch profile created in the Pyrex layer. This eddy is associated with the alignment tolerances between the silicon and Pyrex layers which determines the position at which the inlet duct joins the main channel. Fig. 3b describes the variation in pressure through the inlet region and indicates a high pressure loss corresponding with the restriction in the etched duct, which is suggested by the close proximity of the pressure contours.

With regard to eddy flows being present within the ultrasonic separator during operation, the formation of eddies presents a condition where particle movement cannot be predicted. For example, particles are manipulated by the acoustic

(a)

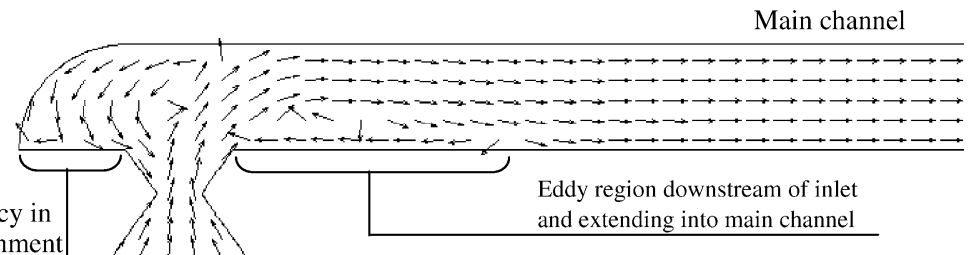

Overhang length determined by accuracy in the Pyrex/silicon alignment and extending into main channel

(b)

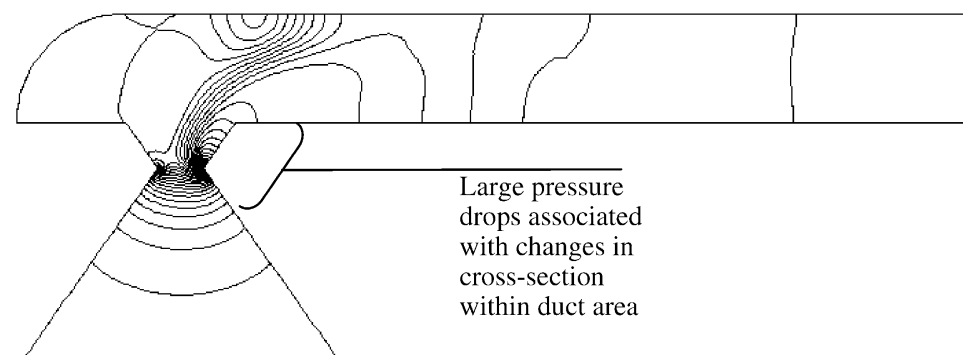

Fig. 3. (a) Vector plot illustrating development of eddy downstream of inlet and (b) associated pressure drop indicated by pressure contours. 
field within the main channel, but the presence of an eddy in this region will distort the laminar flow field and also particle trajectories, which in turn influences the separation efficiency of the device. Additionally, the degree to which particles become trapped within eddies is unknown, but presents doubts over the ability to successfully flush clean the device should it be required for subsequent operations.

\section{Development of CFD model}

In order to study the influence that the microchannel geometry has on the development of eddies, a CFD model is used. This enables a variety of possible geometries to be modelled over a range of flow rates and provides comprehensive visualisation of the flow, an aspect difficult to achieve in the fabricated device.

\subsection{Application of CFD to microfluidic simulation}

Recent studies exist which aim to identify the scale at which classical flow theory and the Navier-Stokes set of governing equations cease to accurately describe fluid flow systems [12-14]. These studies have been occasionally collated, for example, by Judy et al. [15] and Gad-el-Hak [16]. Judy et al. [15] note that deviations from macroscale flow theory have generally been observed for hydraulic diameters below $100 \mu \mathrm{m}$, therefore as the micro-channel dimensions in this work are greater than $100 \mu \mathrm{m}$ this supports the use of the Navier-Stokes equations in this application. Commercial numerical packages based on the Navier-Stokes equations have been applied to the analysis of fluid flow in microfluidic systems, for example, aspects of fluid flow through injector systems incorporating channels of dimensions $200 \mu \mathrm{m}[17,18]$ and micromixer channels down to dimensions of $70 \mu \mathrm{m}$ [19] have been simulated. Therefore, a commercial CFD code, CFX5, has been chosen for the fluid simulation work described in this paper.

The aspect ratio of the fluid channels is typically greater than 20 , therefore to reduce the size of the model and therefore computation time, 2D models are used to simulate the fluid flow through various parts of the device. The 2D models are effectively thin 3D slices along the length of the channel bounded either side by symmetry planes. As the concentration of yeast particles used in experiment is low enough to assume negligible effect on the fluid dynamics, the CFD simulations are further simplified by modelling only the fluid flow and exclude the influence of solid phase particles. A mesh dependence study has been completed based on a simulation of the main channel geometry, essentially a model describing flow between parallel plates known as Poiseuille flow. From this study a mesh refinement level has been selected and results in an average error of less than $1 \%$ when compared to the classical parabolic velocity profile for laminar flow. In addition, mesh adaption is used for selective mesh refinement during the computation. This refines the mesh in regions exhibiting a high velocity or pressure gradient, which usually occur adjacent to wall boundaries and at geometric discontinuities, associated with the formation of eddy flows.

\subsection{Validation of CFD model}

An initial batch of microengineered cells has been produced to investigate the ultrasonic separation capabilities of a micro-engineered device and contains devices of the two different duct geometry designs shown in Fig. 2. These devices were also used to validate the CFD model by measuring and comparing the size of the eddy region downstream of the inlet duct. The eddy region in question is illustrated (Fig. 3) and is chosen as it can be observed in the fabricated devices, although it is appreciated that other unobservable eddies exist within the device. The experimental results taken from the fabricated devices were extracted by studying the movement of particles under the influence of the flow field only (it has been assumed that particles have little influence on the fluid dynamics and are neutrally buoyant). This is achieved by pumping a mixture of water and a low concentration of yeast particles through the device at a known flow rate and using a Motic DMB3 digital biological microscope to record images looking down through the device Pyrex layer. An example image is shown in Fig. 4 which illustrates the eddy formation downstream of the inlet duct, discernible from the laminar stream, and corresponds to the geometry and flow pattern shown in Fig. 3 CFD results are collected by modelling the inlet region of both duct designs and using streamline plots to determine the extent of the eddy region from the leading edge of the main channel.

It has been observed that the flow velocity through the inlet duct is not uniform across the width of the device and is

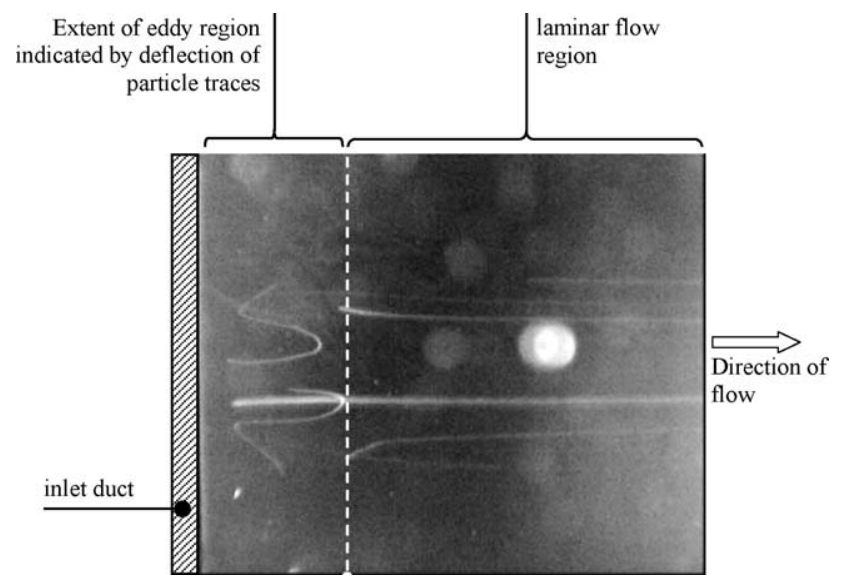

Fig. 4. Image taken whilst looking down through Pyrex layer showing the inlet duct extending across the width of the device. Streamlines created by yeast particles passing along the main channel can be seen. Immediately downstream of the inlet duct some particles are deflected by the eddy region, as can be seen by their convoluted path. To the right of this region particles can be seen following straight streamlines where the laminar flow profile has developed. Video of moving particles gives clearer indication of the extent of the eddy region as compared to still images. 


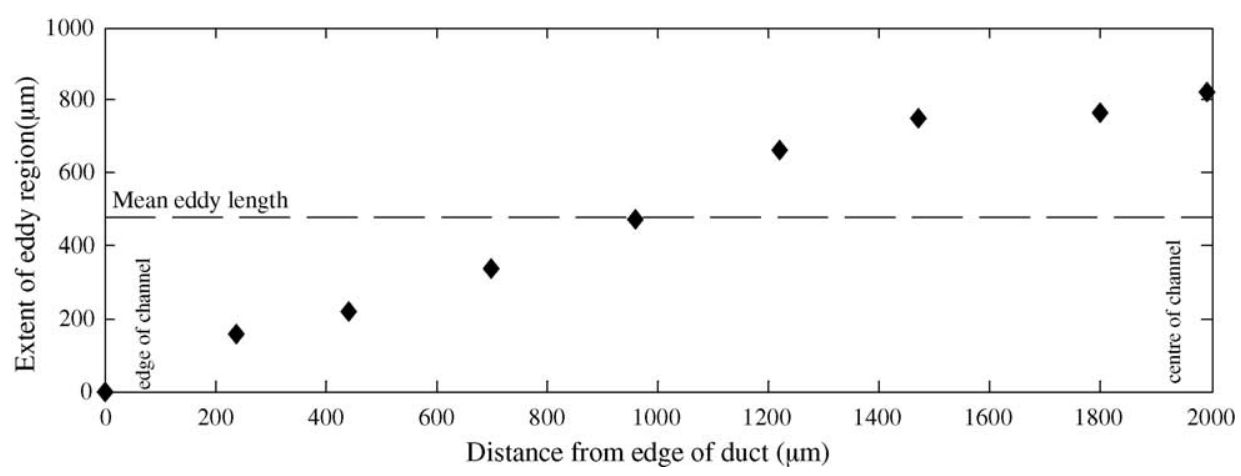

Fig. 5. Variation in eddy size across the width of the main channel and downstream of the double etched inlet duct at a flow rate of $0.3 \mathrm{ml} / \mathrm{s}$.

caused by the manifold geometry beneath the device which issues a narrow jet of fluid towards the centre of the inlet. This causes the size of the associated eddy to vary across the width which is demonstrated in the plot shown in Fig. 5, the data for which is measured experimentally. It can be seen that near the side of the channel the eddy region is small and corresponds to a lower flow velocity, whereas towards the centre of the channel the jet emanating from the manifold creates a larger flow velocity and therefore longer eddy region. At flow rates between 0.1 and $0.3 \mathrm{ml} / \mathrm{s}$ the calculated mean eddy size is on average approximately 0.5 times that of the maximum (centre) eddy length and is indicated on the plot. Therefore, to simplify the experimental work, the maximum eddy size only has been measured and adjusted by a factor of 0.5 to take into account the non-uniform flow and to record a mean eddy length.

In Fig. 6, the size of the eddy region is plotted as a function of flow rate and demonstrates that eddy size is strongly related to the fluid flow rate. An initial inspection shows that the CFD results underestimate the length of the eddy region, however they both show that the double-etched geometry produces a

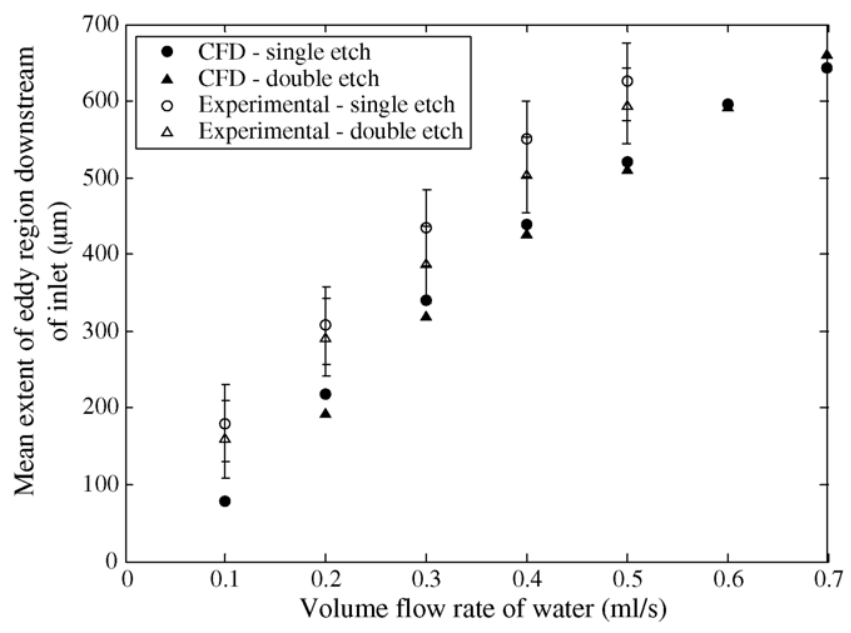

Fig. 6. Extent of eddy region downstream of inlet duct for both single and double etched geometry over a range of flow rates and plotted for both CFD and experimental results. The measurements are based on two sets of experiments and error bars of $\pm 50 \mu \mathrm{m}$ have been added, reflecting the perceived accuracy of the estimates. slightly smaller eddy region compared to the single etch geometry up to a flow rate of $0.5 \mathrm{ml} / \mathrm{s}$. The difference between experimental and CFD results can be explained by the following: (a) in general the exact conditions at the inlet boundary of the CFD model are unknown although a parabolic profile has been assumed; (b) pulses produced by the peristaltic pumps have been observed to cause fluctuations in the flow rate and therefore variations in the eddy size, although it has not been possible to quantify these variations; (c) as demonstrated in Fig. 5, the flow through the device is not uniform across its width and suggests that a more computationally expensive 3D CFD would be more appropriate; (d) a buildup of particles can reduce the duct size and encourages more significant flow separation and a larger eddy, although the device was flushed frequently during experimental work.

In general this exercise demonstrates that a 2D CFD model provides a valuable method to predict the location and scale of eddy formation within microfluidic channels, in this case for ducts incorporating a minimum dimension of $160 \mu \mathrm{m}$. However, by extending the complexity of the simulation to include transient and 3D aspects of the flow, it is anticipated that a more accurate representation of the flow can be generated, should this be required.

\section{Geometric study}

\subsection{Introduction}

The geometric study aims to improve the duct design by restricting the onset of eddies and reducing pressure drops. As eddy regions are typically caused by discontinuities found in the duct geometry, an initial visual inspection can be used to identify these features. The visual inspection covers a range of possible geometries created by the anisotropic etch process. This approach serves to reduce the problem to a few geometric designs, which are then each investigated more thoroughly within CFD simulations.

\subsection{Qualitative study}

Eddy regions occur when the flow becomes separated from the wall. Within a fluid network this occurs in areas where 


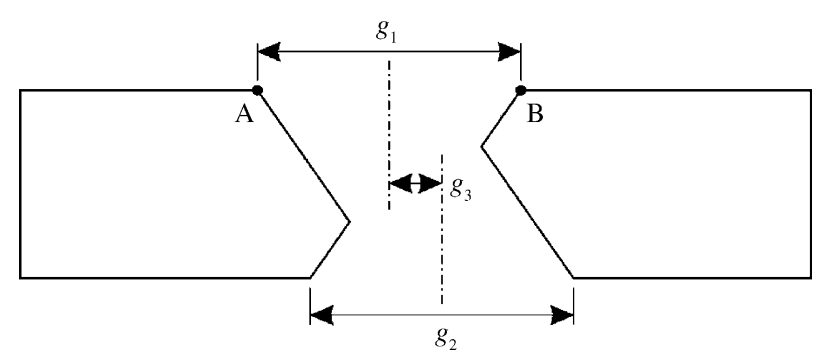

Fig. 7. Parameters describing silicon etched duct geometry; for top and bottom etch masks, $g_{1}$ and $g_{2}$ describe respective widths, and $g_{3}$ the relative offset.

the cross-section changes such as in diffusive channels and at discontinuities and sharp edges in the wall geometry. For example, an anisotropic etch such as the single-etched duct inherently creates a diffusive duct with an inclusive angle of $109^{\circ}$, recognised to result in significant losses as compared to the optimum angle of $6-7^{\circ}$ [11]. Also, the separator device is formed from multiple wafer layers and misalignment between these can introduce sharp edges and sudden expansions or reductions in the fluid channel size. For example, where a single-etched duct acts as a convergent channel (inlet duct in Fig. 2a) as the fluid enters the main channel the fluid is subject to an extreme expansion, although no eddy is necessarily induced in the duct itself.

For the reasons discussed above, the study concentrates on double-etched ducts and in order to efficiently cover a range of possible duct geometries a parametric approach is used. This analysis assumes an anisotropic wet silicon etch such as $\mathrm{KOH}$, where Fig. 7 illustrates the parameters used to describe the resulting etched duct geometry with the direction of flow either way through the duct. Parameters $g_{1}$ and $g_{2}$ describe the width of the top and bottom etch masks and subsequent etched areas, whereas $g_{3}$ describes the distance between the centre points of the top and bottom etch areas, effectively representing their offset and the degree to which the duct is skewed.

For the purposes of the geometric study it has been assumed that the inlet and outlet 2 ducts are positioned at the extreme ends of the main channel, i.e. there are no redundant areas at the ends of the main channel. This is designed to discourage eddy formation either end of the main channel such as that demonstrated in Fig. 3a, near the inlet duct. However, this assumes a fabrication method such that high accuracy is applied both to the alignment of the Pyrex upon the silicon layer and to the isotropic etching of the Pyrex which determines the length of the main channel.

By classifying geometry in terms of the parameters $g_{1}-g_{3}$, Fig. 8 illustrates the basic geometry groups used for the study. Again, fluid flows either way through the duct with the main channel flow passing either from the left or right.

Considering the selection of geometries displayed in Fig. 8, a discontinuity cannot be avoided where fluid passes to the main channel and beyond the silicon layer (point A or B indicated in Fig. 7). However, only the geometry type shown

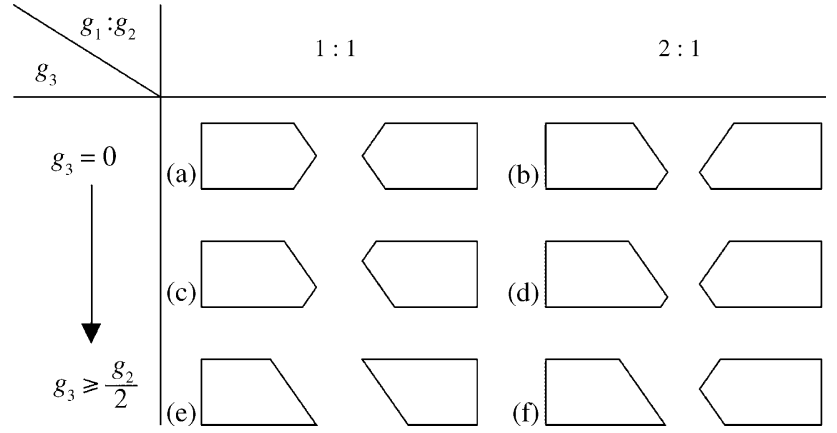

Fig. 8. Basic duct geometry groups.

in Fig. 8e is unlikely to encourage eddy formation within the duct area itself; all other geometries contain discontinuities and a divergent element. It is therefore this parallel sided duct geometry which shall be investigated further.

\subsection{CFD study}

Simple inspection has eliminated geometries which have a significant expansion or contraction. However, more insight is gained from CFD simulations investigating dimensions and the robustness of the flow pattern to small inaccuracies in dimensions and mask alignment. This is achieved by completing a series of simulations of the flow through both the inlet and outlet ducts, investigating the influence of dimensions $g_{1}$ (where $g_{2}=g_{1}$ ) and also the influence of small etch mask inaccuracies which create the geometry illustrated in Fig. 8f. The key observations are as follows and are based on CFD simulations using a flow rate of $0.5 \mathrm{ml} / \mathrm{s}$.
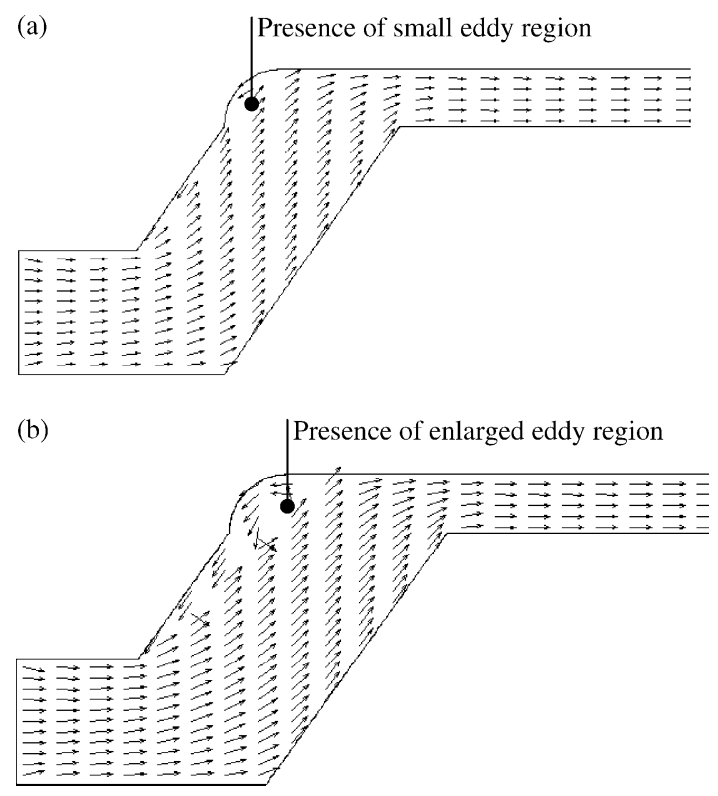

Fig. 9. Vector plots of inlet duct showing the affect of duct width on eddy region size for (a) minimum duct width and (b) an increased duct width of $150 \mu \mathrm{m}$. 


\subsubsection{Duct width}

Over a complete series of simulations, an optimum design is observed when subsequent sections of the flow path remain constant or gradually reduce in cross-sectional size, replicating the effect of a convergent duct and ensuring that flow remains adhered to the wall. An example of this is shown in Fig. 9 which demonstrates that as the width of the parallel sided inlet duct is increased and becomes larger than the preceding channel section, an eddy region is created and expands. Note that this is only of significant advantage in the inlet region of the separator device as only the inlet duct leads to the relatively narrow main channel.

\subsubsection{Error/offset in masks}

Any errors or offset in the double sided alignment process used to align the etch masks on either side of the silicon wafer will potentially introduce a discontinuity. Such discontinuities generally encourage an eddy, the eddy size being related to the magnitude of the error/offset. Fig. 10 depicts the outlet 2 duct shows one example of this where an eddy region forms within the area created by the curved isotropic etch profile within the Pyrex layer and the chamfer within the anisotropically etch silicon duct. However, the flow through outlet 1 is not influenced by the Pyrex etch profile and is less sensitive to double sided alignment inaccuracies. Fig. 11 shows that an eddy only forms within the main channel above the duct at a high degree of alignment inaccuracy where a large chamfer is present in the duct. This is unlikely to occur since the double sided alignment process is typically accurate to $\pm 5 \mu \mathrm{m}$.

\section{Device fabrication and test}

Parallel ducts have been used within a new batch of devices, where Fig. 12 shows an example of the outlet 2 region and is taken from a cross-sectioned device. The potential chamfering of the duct edges produced by errors in alignment has not occurred. However, the alignment of the
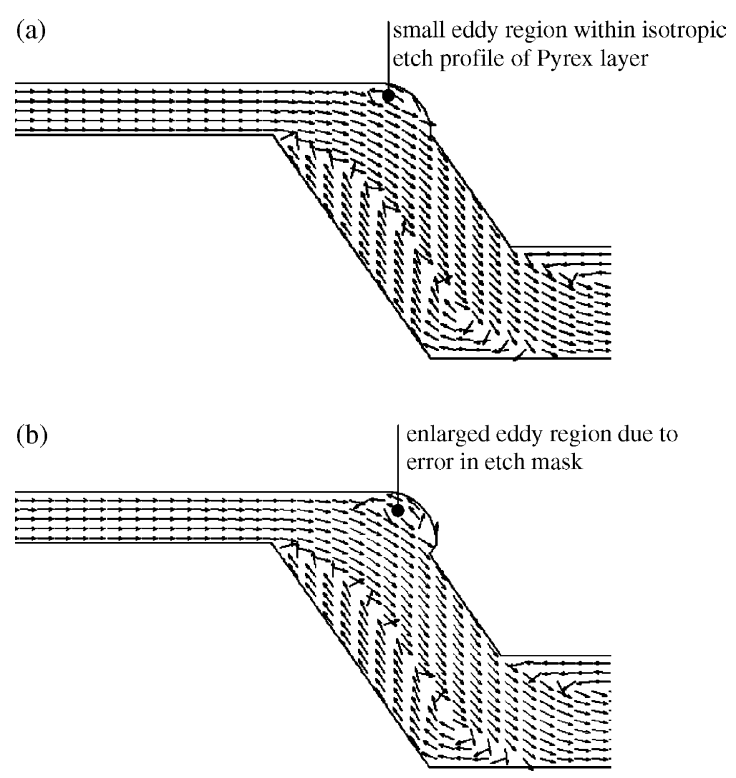

Fig. 10. Vector plots of outlet 2 showing effect of error/offset in etch mask for (a) no offset and (b) top etch increased by $50 \mu \mathrm{m}$.

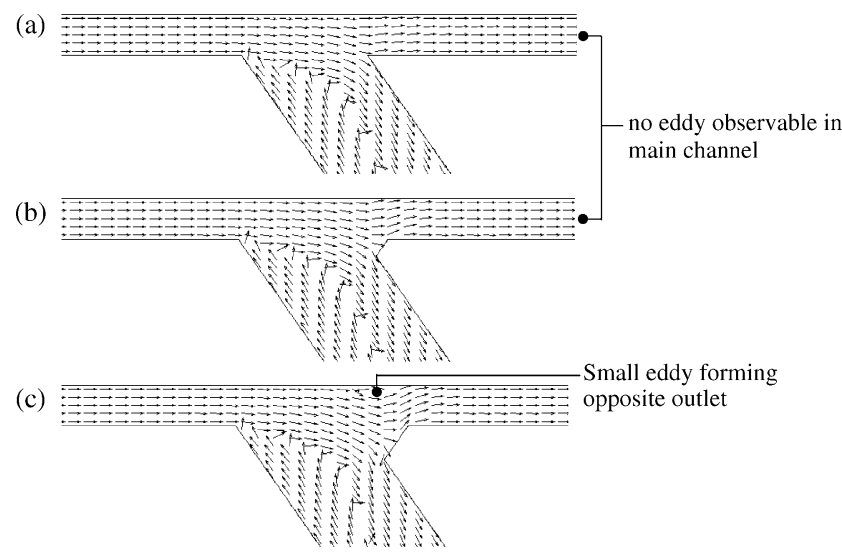

Fig. 11. Vector plots of outlet 1 showing effect of error/offset in etch mask for (a) no offset and (b) top etch increased by $50 \mu \mathrm{m}$ (c) top etch increased by $100 \mu \mathrm{m}$.

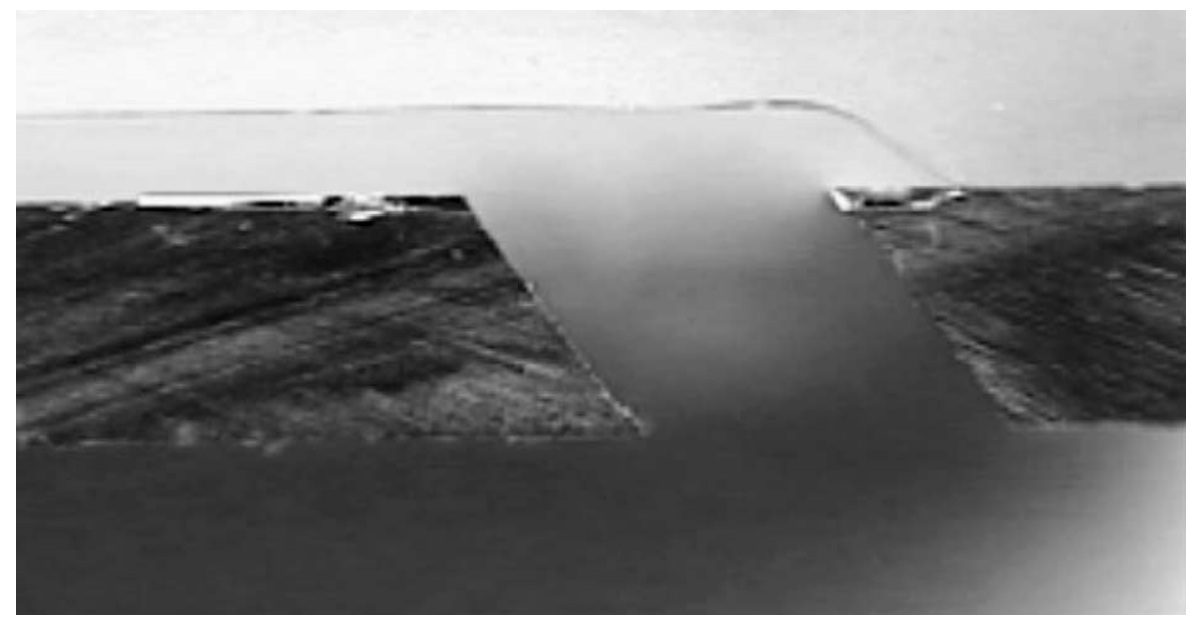

Fig. 12. Cross-section of etched device incorporating revised geometry. 


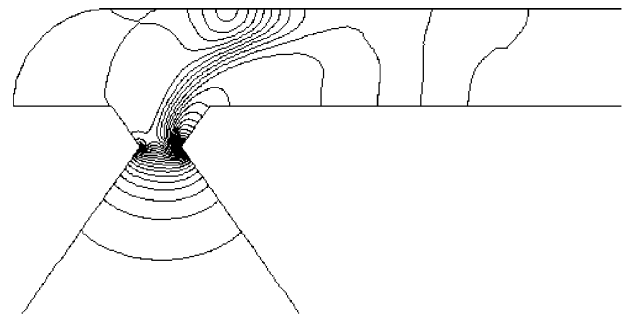

(b)

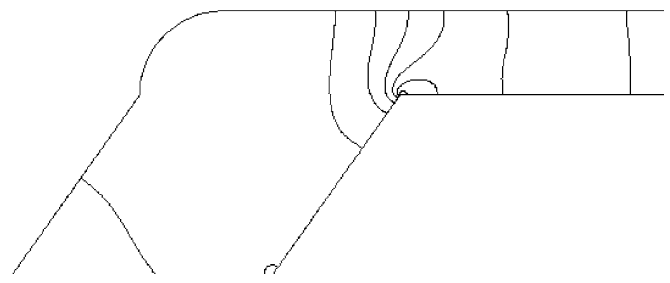

Fig. 13. Pressure distributions within (a) double-etched and (b) parallel sided duct geometry at a flow rate of $0.5 \mathrm{ml} / \mathrm{s}$ where contours plotted at ca. $35 \mathrm{~Pa}$ intervals.

Pyrex and silicon wafers, which was performed by eye, means that the outlet duct is not aligned exactly with the end of the main channel. The use of an optically aligned anodic bonder would produce more satisfactory results by reducing this overhang.

The device is tested using the same method described in Section 3 and no eddy downstream of the inlet is observed up to flows of $0.6 \mathrm{ml} / \mathrm{s}$, beyond which the flow pattern is difficult to discern. This level of eddy suppression is significant when compared to the results recorded in Fig. 6 for the original, more angular geometry where eddies downstream of the inlet exist at a much lower flow rate of $0.1 \mathrm{ml} / \mathrm{s}$ and possibly below this value. The only drawback of the parallel duct is the imposition of a minimum channel width which is based upon the silicon wafer thickness.

Fig. 13 shows the pressure distribution in both the doubleetched and parallel sided inlet duct geometry, where the large pressure drops seen within the double-etched geometry are not observed within the new parallel geometry where the pressure contours are much more widely and evenly spaced. It is accepted that eddy formation, and possibly pressure losses, are still likely to be significant within the outlet ducts, although more careful design of the downstream fluid network would be beneficial.

\section{Conclusion}

The anisotropically etched silicon duct geometries presented in this paper reduce eddy development in the ultrasonic separator and the associated fluid losses. CFD and experimental results demonstrate that this has been most successful in the main channel of the device where highly laminar flow is required for predictable operation of the device in manipulating particles. In one case, problematic eddy formation has been eliminated within a range of flow rates at least six times larger than that of the original geometry. Eddy flows within the ducts, which act as potential capture sites for particles and contaminants, have been investigated and limited by the use of parallel sided ducts. Further work is required to improve aspects of the outlet ducts where the flow field is highly dependent upon the downstream fluid network.

The reduced pressure drops, associated with geometry resulting in the smaller eddy flows, reduce the total pressure drop across the device. This is useful where pumping methods are low power devices and resistance offered by the fluid system needs to be minimised.

\section{Acknowledgements}

This work is funded by EPSRC grant GR/R13333/01. The authors gratefully acknowledge the funding and support from Dstl and Porvair plc.

\section{References}

[1] C.M. Cousins, P. Holownia, J.S. Hawkes, C.P. Price, P. Keay, W.T. Coakley, Clarification of plasma from whole human blood using ultrasound, Ultrasonics 38 (2000) 654-656.

[2] D.A. Johnson, D.L. Feke, Methodology for fractionating suspended particles using ultrasonic standing wave and divided flow fields, Sep. Technol. 5 (1995) 251-258.

[3] H. Bohm, L.G. Briarty, K.C. Lowe, J.B. Power, E. Benes, M.R. Davey, Quantification of a novel h-shaped ultrasonic resonator for separation of biomaterials under terrestrial gravity and microgravity conditions, Biotechnol. Bioeng. 82 (2003) 74-85.

[4] J.J. Hawkes, W.T. Coakley, Force field particle filter, combining ultrasound standing waves and laminar flow, Sens. Actuators B Chem. 75 (2001) 213-222.

[5] N.R. Harris, M. Hill, S. Beeby, Y. Shen, N.M. White, J.J. Hawkes, W.T. Coakley, A silicon microfluidic ultrasonic separator, Sens. Actuators B Chem. 95 (2003) 425-434.

[6] M. Hill, N. Harris, R. Townsend, N. White, S. Beeby, Separation of particles from a fluid using a microfabricated, ultrasonic device, in: Proceedings of the Fifth World Congress on Ultrasonics, Paris, France, 2003.

[7] M. Hill, Y.J. Shen, J.J. Hawkes, Modelling of layered resonators for ultrasonic separation, Ultrasonics 40 (2002) 385-392.

[8] J.J. Hawkes, W.T. Coakley, M. Groschl, E. Benes, S. Armstrong, P.J. Tasker, H. Nowotny, Single half-wavelength ultrasonic particle filter: predictions of the transfer matrix multilayer resonator model and experimental filtration results, J. Acoustic. Soc. Am. 111 (2002) $1259-1266$.

[9] R.J. Townsend, M. Hill, N.R. Harris, N.M. White, Modelling of particle paths passing through an ultrasonic standing wave, Ultrasonics 42 (2004) 319-324.

[10] F.M. White, Fluid Mechanics, McGraw-Hill, 1999.

[11] J.F. Douglas, J.M. Gasiorek, J.A. Swaffield, Fluid Mechanics, Prentice Hall, Harlow, 2000.

[12] J. Pfahler, J. Harley, H. Bau, J. Zemel, Liquid transport in micron and submicron channels, Sens. Actuators A Phys. 22 (1990) 431-434.

[13] X.F. Peng, G.P. Peterson, Convective heat transfer and flow friction for water flow in microchannel structures, Int. J. Heat Mass Transfer 39 (1996) 2599-2608.

[14] G.M. Mala, D.Q. Li, Flow characteristics of water in microtubes, Int. J. Heat Fluid Flow 20 (1999) 142-148. 
[15] J. Judy, D. Maynes, B.W. Webb, Characterization of frictional pressure drop for liquid flows through microchannels, Int. J. Heat Mass Transfer 45 (2002) 3477-3489.

[16] M. Gad-el-Hak, The MEMS Handbook, CRC, Boca Raton, 2001.

[17] X.X. Bai, J. Josserand, H. Jensen, J.S. Rossier, H.H. Girault, Finite element simulation of pinched pressure-driven flow injection in microchannels, Anal. Chem. 74 (2002) 6205-6215.

[18] E.B. van Akker, M. Bos, W.E. van der Linden, Convection and diffusion in a micro-flow injection system, Anal. Chim. Acta 373 (1998) 227-239.

[19] S. Ehlers, K. Elgeti, T. Menzel, G. Wiessmeier, Mixing in the offstream of a microchannel system, Chem. Eng. Process. 39 (2000) 291-298.

\section{Biographies}

R.J. Townsend graduated in 2000 from the University of Southampton with an MEng in mechanical engineering and is now studying for a $\mathrm{PhD}$ within the Electro-mechanical Research Group at Southampton. Her work involves the numerical simulation of particles under the influence of acoustic forces and other aspects of microfluidic device simulation. She is an associate member of the IMechE.

M. Hill is Reader in Electromechanical Systems in the School of Engineering Sciences, Southampton University. He graduated in 1985 from the Institute of Sound and Vibration Research, was appointed lecturer in 1990 and has research interests in acoustics (particularly ultrasonics) and transducers. His work has led to more than 80 publications, he is a chartered engineer, a member of the Institute of Measurement and Control, and a member of the Institute of Acoustics.

N.R. Harris graduated in 1988 from the University of Bath and was awarded a PhD in 1997 from the University of Southampton, on the use of ultrasound for communication purposes. His research interests include thick-film sensors and surface acoustic wave devices, microengineering, and sensor interfacing. He is now a lecturer based in the Electronics and Computer Science Department at Southampton. He is a member of the IEE and a chartered engineer.

N.M. White is a professor of intelligent sensor systems within the School of Electronics and Computer Science at the University of Southamp- ton and also director of the Institute of Transducer Technology. He was awarded a $\mathrm{PhD}$ in 1988 for a thesis on the application of thick-film piezoresistors for load cells. Professor N.M. White was appointed as lecturer in 1990, senior lecturer in 1999, reader in 2000 and currently holds a Personal Chair. He has published extensively in the area of thick-film sensors and intelligent instrumentation and is author or co-author of over one hundred scientific publications. He is a fellow of the Institute of Physics, a chartered engineer and a senior member of the IEEE and has served of several committees in various professional bodies.

S.P. Beeby graduated from the University of Portsmouth in 1992 with BEng (Hons) in mechanical engineering. He obtained a $\mathrm{PhD}$ from the University of Southampton in 1998. After his $\mathrm{PhD}$ he obtained industrial funding for a follow up project to develop a resonant differential pressure sensor. He has since been awarded a prestigious EPSRC Advanced Research fellowship to continue his research into active thick film material development and their combination with micromachined devices. His other research interests include energy harvesting for remote wireless sensor networks and he is the principal investigator at Southampton on an EU funded STREP project entitled 'Vibration Energy Scavenging (VIBES)'. He is also interested in human biometric systems. He has over 90 publications in the field including in learned journals and presented at conferences and colloquia. He is co-author of a recently completed book entitled 'MEMS Mechanical Sensors' published by Artech House. He is a member of the EPSRC peer review College, reviewer for numerous journal publications, a Chartered Engineer and Chartered Physicist and has provided consultancy services to several companies.

R.J.K. Wood has recently been promoted to Professor of Surface Engineering and Tribology in the Materials and Surface Engineering Research Group and is Director of the Institute of Research for Industry within the School of Engineering Sciences at the University of Southampton. He obtained a PhD in erosion-corrosion research from University of Southampton in 1987 and has over 15 years of research experience in the field of tribology and surface engineering. He has spent several years at BP Research but returned to Southampton in 1993 to re-establish surface engineering/tribology research. His group has three postdoctoral research fellows and six research students. Professor R.J.K. Wood has published over 80 papers in tribology research and related areas and has attracted over $£ 2.5 \mathrm{M}$ research funding to this area. He has been a member of the EPSRC peer review College since 2000 and a chartered engineer and a member of the Institution of Mechanical Engineers and a member of the Institute of Corrosion since 1992. 